\title{
A Simple Continuous Wavelet Transform Method for Detection of Rolling Element Bearing Faults and its Comparison with Envelope Detection
}

\author{
Sunil Tyagi ${ }^{1}$, SK Panigrahi ${ }^{2}$ \\ ${ }^{1,2}$ Department of Mechanical Engineering, Defense Institute of Advance Technology, Girinagar, Pune - 411025, India
}

\begin{abstract}
Envelope Detection (ED) is traditionally used to identify the rolling element bearing faults. ED involves carrying considerable signal processing. In past few years Wavelet Analysis (WA) has been widely used an alternative tool for detection of machinery fault diagnosis as WA is able to provide multi-resolution analysis and give both frequency and time information suitable to analyse non-stationery signals such as vibration signal from bearing. This paper presents a very simple Wavelet analysis technique using Continuous Wavelet Transform (CWT) that does not require complex signal processing. A comparative study of effectiveness of ED and CWT method for bearing fault diagnosis is presented in this paper and it is shown with help of experimental results that the proposed simple CWT based method is better fault diagnostic tool for bearing fault identification.
\end{abstract}

Keywords: Ball bearing, Continuous Wavelet Transform (CWT), Envelope Detection (ED), Fault Diagnosis, High Frequency Resonant Technique (HFRT), Hilbert Transform, Rolling element bearing, Machinery Vibration

\section{Introduction}

Rolling element bearings or ball/roller bearings are most common machine component of any rotating machinery used in industry. Rolling element bearings endure heavy loads under industrial operating conditions and structural faults, such as wear, pitting, or spall may occur after a long period of running [1]. Components that often fail in rolling element bearing are outer-race, inner-race and the ball. The bearing defects generate a series of impact vibrations every time a running ball passes over the surfaces of the defects. These impacts recur at Bearing Characteristic Frequencies (BCF), which are estimated based on the running speed of shaft, the geometry of the bearing, and the location of the defect [2]. Because the impact generated by a bearing fault distributes its energy over wide frequency range thus the $\mathrm{BCF}$ has relatively low energy, it is often overwhelmed by high-energy noise and vibrations generated from other macro structural components.

Traditional frequency domain analysis or time domain analysis of raw vibration signal using contemporary techniques is unable to detect the ball bearing faults. Hence,
Envelope Detection (ED) also known as High Frequency Resonant Technique (HFRT) has been used for bearing fault detection because the envelope signals provide much more diagnostic information than the raw signals [3, 4]. The impact vibrations are difficult to be identified in low frequency range due to their low energy and interference from other vibration sources, the usual practice is to view these micro-structural vibrations at the bearing resonance frequency range. The modulated amplitudes of repetitive impacts are often excited at the bearing structural resonance frequency. Hence, the amplitude demodulation provided by ED allows the detection of localized detects [1].

Although, ED is an effective technique to detect faults of rolling element bearings but it performance deteriorates if band-pass window is not carefully selected $[1,3]$. Recently, wavelet transform has been applied for rolling element bearing fault diagnosis [5 - 8] as WA is able to generate time-frequency distribution diagram and it provides multiresolution analysis that is especially useful to analyse nonstationary signals such as vibration signal emanating from ball-bearings [9].

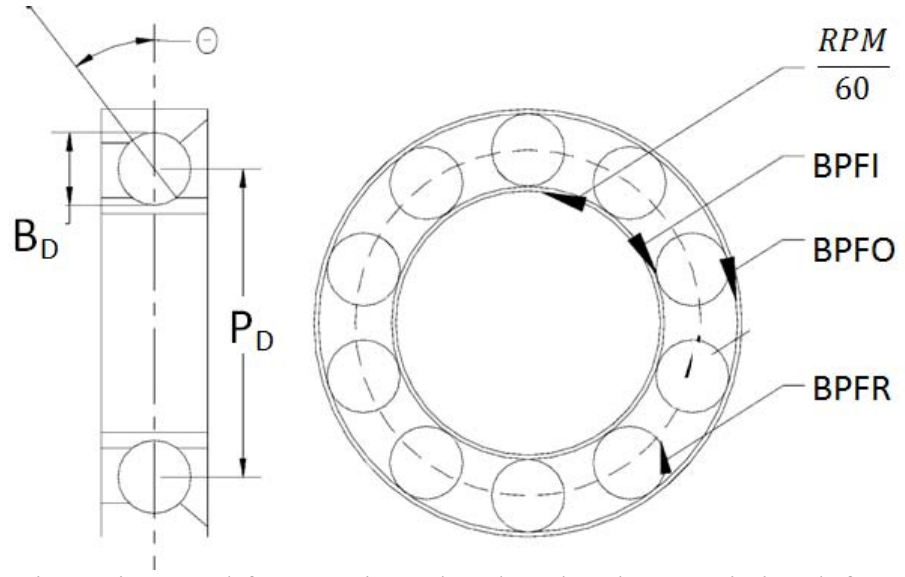

Figure 1: Dimensions and frequencies related to the characteristics defect frequencies

Volume 6 Issue 3, March 2017 www.ijsr.net

Licensed Under Creative Commons Attribution CC BY 


\section{International Journal of Science and Research (IJSR) \\ ISSN (Online): 2319-7064}

Index Copernicus Value (2015): 78.96 | Impact Factor (2015): 6.391

\section{Bearing Defect and Characteristic Frequencies}

Operation of bearing with defects causes rolling element passed over localized discreet defect on outer or inner race of bearing. This causes an abrupt change in the contact stresses at the edge. This abrupt change in contact stresses generate a high-level short duration impulsive force that excites the bearings structural resonance resulting in high frequency damped oscillations that are periodically repeated at the frequency of rolling element passing over the defect known as bearing characteristic defect frequency.

The bearing characteristic defect frequencies as shown in Fig. 1 are calculated from geometric dimensions of the bearing, number of balls and shaft frequency as given below:

Ball Pass Frequency Outer Race

$$
B P F O=\frac{n}{2} \frac{R P M}{60}\left(1-\frac{B_{d}}{P_{d}} \cos \emptyset\right)
$$

Ball Pass Frequency Inner Race

$$
B P F I=\frac{n}{2} \frac{R P M}{60}\left(1+\frac{B_{d}}{P_{d}} \cos \emptyset\right)
$$

Ball Pass Frequency Rolling Element

$$
B P F R=\frac{P_{d}}{2 B_{d}} \frac{R P M}{60}\left[1-\left(\frac{B_{d}}{P_{d}}\right)^{2} \cos ^{2} \emptyset\right]
$$

Where $\boldsymbol{B}_{\boldsymbol{d}}$ is Ball diameter, $\boldsymbol{P}_{\boldsymbol{d}}$ is Pitch diameter, $\boldsymbol{n}$ is number of balls and $\boldsymbol{\varphi}$ is the contact angle [2].

\section{Envelope Detection}

Fundamental to the ED is the concept that each time a defect in a rolling element bearing makes contact under load with another surface in the bearing, an impulse is generated. This impulse is of extremely short duration compared with the interval between impulses, and its energy is distributed at a very low level over a wide range of frequencies. It is this wide distribution of energy, which makes bearing defects so difficult to detect by conventional spectrum analysis in the presence of vibration from other machine elements. Fortunately, the impact usually excites a resonance in the system at a much higher frequency than the vibration generated by the other machine elements, with the result that some of the energy is concentrated into a narrow band near bearing resonance frequency. As a result of bearing excitation repeated burst of high frequency vibrations are produced, which is more readily detected. Take for example the bearing that is developing a crack in its outer race. Each time a ball passes over the crack, it creates a high-frequency burst of vibration, with each burst lasting for a very short time. In the simple spectra of this signal one would expect a peak at BPFO instead we get high frequency — mystack" because of excitation of bearing structural resonance. The signal produced is an amplitude-modulated signal with bearing structural resonance frequency as the carrier frequency and the modulation of amplitude is by the BCF (message signal). Envelope Detection, which the technique for amplitude demodulation is always, used to find out the repeated impulse type signals. The ED involves three main steps.

The Process On the "typical" time waveform shown in Fig 2 (a), the large wave indicates a large low-frequency component (perhaps due to misalignment, or unbalance). On top of the low-frequency component are superimposed the bursts of high frequency vibrations from the impacts due to bearing defect.

First step is to apply a band-pass filter, which removes the large low-frequency components as well as the high frequency noise only the burst of high frequency vibrations remains as shown in Fig. 2 (b). Next, we trace an "envelope" around the bursts in the waveform (Fig. 2 (c)) to identify the impact events as repetitions of the same fault. In third step, FFT of this enveloped signal is taken, to obtain a frequency spectrum. It now clearly presents the BPFO peaks (and harmonics) as shown is Fig. 2 (d).

The bearing structural resonance frequency is selected as the central frequency of the bandpass filter. Traditionally, impact tests are carried out on bearing to identify the resonant frequency. However, impact tests are not a necessity; the resonant frequency can be identified from inspection of the unfiltered signal's spectrum [2]. There are different ways to extract the envelope, traditionally bandpass filtering, rectifying and lowpass filtering is used to carry out the demodulation. However, Hilbert transform has also been used very effectively for ED [3]. In present work, Hilbert Transform is used to extract the envelope.

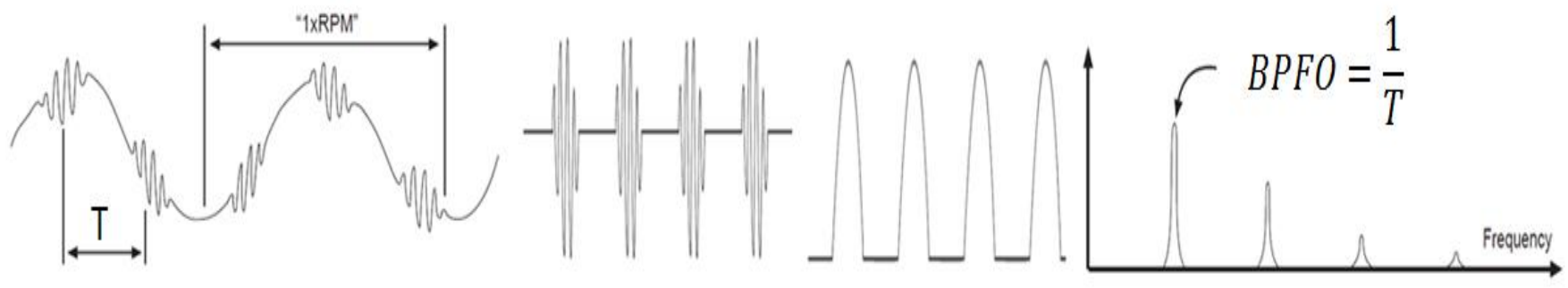
(a) Unfiltered Time domain Signal
(b) Band passed Signal
(c) Envelope of Band passed Signal

Figure 2: Envelope detection process 


\section{International Journal of Science and Research (IJSR)

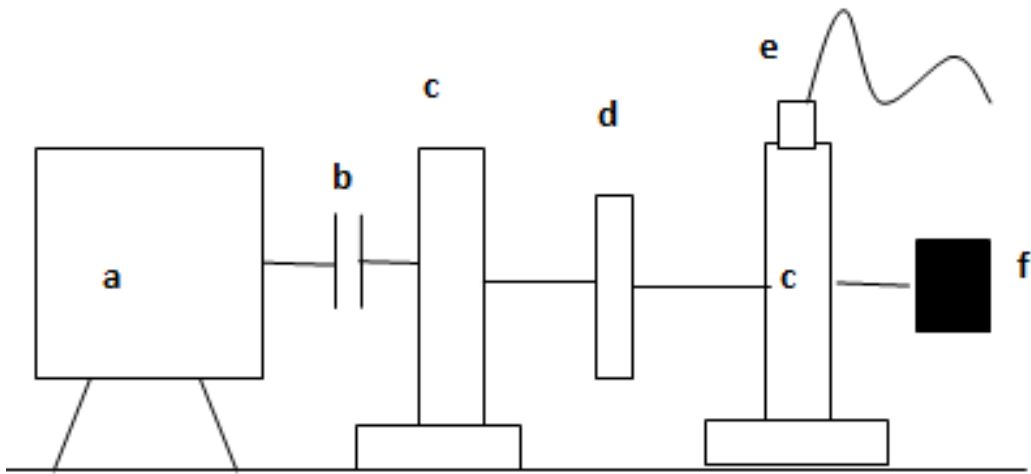

Figure 3: Test rig (a) Variable speed motor (b) Flexible coupling (c) Bearing Housing (d) Load (e) Accelerometer (f) Magnetic break

Hilbert transform The Hilbert Transform is a 90-degree phase shifter [10]. All negative frequencies of a signal get a $+90^{\circ}$ phase shift and all positive frequencies get a $-90^{\circ}$ phase shift. The frequency response function of a Hilbert Transformer has the following property.

$$
H(\omega)=\left\{\begin{array}{l}
-j \text { for } \omega>0 \\
j \text { for } \omega<0
\end{array}\right.
$$

Hilbert transform in Time Domain for a given a signal $\mathrm{x}(\mathrm{t})$, is defined as

$$
\hat{x}(t)=\frac{1}{\pi} \int_{-\infty}^{+\infty} \frac{x(t)}{t-\tau} d \tau(5)
$$

It is the convolution of the signal $x(t)$ with function $1 / \pi \mathrm{t}$, which is the impulse response function of the Hilbert Transformer. The phase shifted and original signals are summed up to obtain an analytic signal $\mathrm{x}_{+}(\mathrm{t})$ defined as follows.

$$
\mathrm{x}_{+}(\mathrm{t})=\mathrm{x}(\mathrm{t})+\mathrm{j} \hat{x}(\mathrm{t})
$$

The Hilbert transform is typically implemented as an FIR filter so the original signal must be delayed to match the group delay of the Hilbert transform. This process can be followed by taking the absolute value of analytic function to generate the envelope $\mathrm{v}(\mathrm{t})$.

$$
v(t)=\sqrt{x^{2}(t)+x_{+}{ }^{2}(t)}
$$

\section{The Continuous Wavelet Transform and Wavelet Analysis}

The wavelet transform is a tool that cuts up data, functions or operators into different frequency components, and then studies each component with solution matched to its scale. The use of wavelet transform is appropriate to analyze nonstationary signal since it gives the information about the signal both in frequency and time domains [11]. The idea behind the CWT is to scale and translate the basic wavelet shape by very small steps in relation to a continuous signal and to compute the wavelet coefficient at each step Let $x(t)$ be the signal. The continuous wavelet transform (CWT) of $x(t)$ is defined as:

$$
W_{\psi}(\tau, s)=\int_{-\infty}^{+\infty} x(t) \cdot \psi_{\tau, s}^{*}(t) d t(8)
$$

where $\psi_{\tau, s}^{*}$ is the conjugate of $\psi_{\tau, g}(t)$ which is the shifted and scaled form of the transforming function, named as - rother wavelet", which is defined as:

$$
\Psi_{\tau, s}(\mathrm{t})=\frac{1}{\sqrt{s}} \psi\left(\frac{\mathrm{t}-\tau}{s}\right)
$$

The transformed signal obtained in equation 8 above is a function of $\mathbf{s}$ and $\tau$, which are the scale and translation parameters respectively.

The mother wavelet is a prototype for generating the other wavelet (window) functions. The scale parameter performs scaling operation on the mother wavelet i.e. stretching and compressing the mother wavelet function, which in turn can be used to represent the signal in different frequency range. Each scale would represent a frequency band. Scale may roughly be considered as inverse of frequency as low scales represents high frequency band and high scales represents low frequencies. The term translation corresponds to time information in the transform domain; it shifts the wavelet along the time axis to capture the time information contained in the signal.

There are many types of wavelet functions available for different purposes, such as the Harr, Dabechies, Gaussian, Meyer Mexican Hat, and Morlet functions. The admissibility condition for each type of wavelet should satisfy the admissibility condition $[\mathbf{1 2}]$ as below:

$$
0<\int_{-\infty}^{+\infty} \frac{|\Psi(\omega)|^{2}}{\omega}<+\infty(10)
$$

where $\Psi(\varpi)$ is the FFT of $\psi(t)$. From Eq. 10 it follows that $\Psi(\varpi)=0$ for $\omega=0$, that is the wavelet function $\psi(t)$ has a zero average value, meaning that $\int_{-\infty}^{+\infty} \Psi(t)=0$. This is the reason why $\psi(t)$ must be oscillatory. In other words, it must be a wave. 
International Journal of Science and Research (IJSR)

ISSN (Online): 2319-7064

Index Copernicus Value (2015): 78.96 | Impact Factor (2015): 6.391

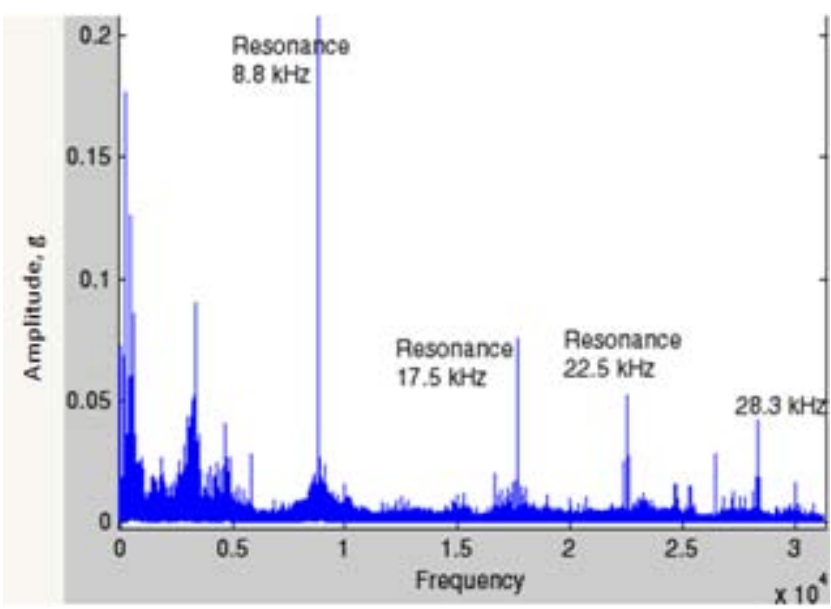

Figure 4: Spectrum of Bearing with outer race defect

Table 1: Calculated BCF for different faults

\begin{tabular}{|l|l|l|l|}
\hline & BPFO & BPFI & BPFR \\
\hline BCF for Shaft frequency (Hz) & 86.6 & 144.3 & 107.9 \\
\hline Time Interval of Impacts (ms) & 11.6 & 6.93 & 9.27 \\
\hline Samples between impacts (Nos.) & 723 & 433 & 579 \\
\hline
\end{tabular}

Generally, Continuous Wavelet Transform is preferable in situations where minute resolution is required, as the resolution of CWT is higher compared to the Discreet type of Wavelet Transform. The Meyer wavelet is an orthogonal wavelet proposed by Yves Meyer. It is infinitely differentiable with infinite support. In this study, a continuous type of Meyer wavelet is used.

\section{Experimental Setup}

The test rig shown in Fig. 3 was composed of a variable speed AC motor driving a shaft rotor assembly through flexible couplers; shafts were rested on two ball bearings. A rotor was used for balancing. The bearings under analysis (type MB 204) were placed at load end side for ease of replacement. The load on the system can be adjusted by a

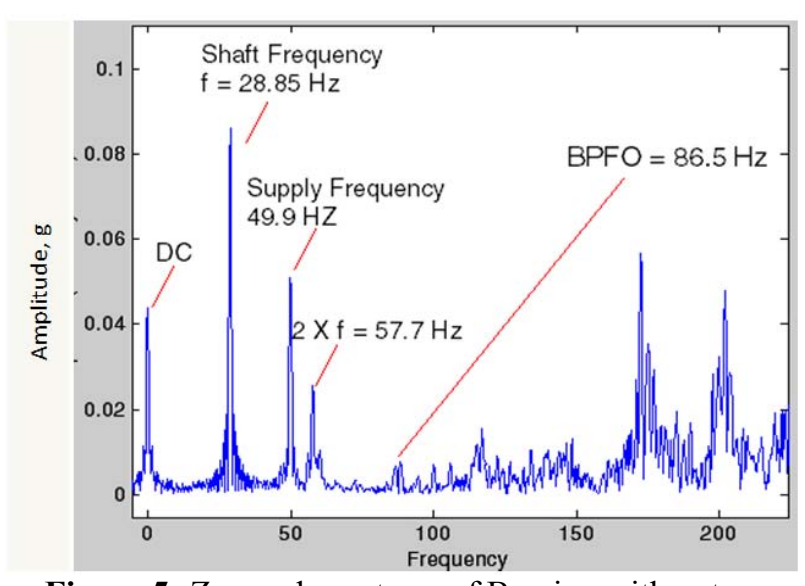

Figure 5: Zoomed spectrum of Bearing with outer race defect

manually adjustable magnetic brake, which was driven via a belt drive. Three defective bearings under analysis were mounted on free end side consequently and they were introduced with only one type of fault at only one location. Fault measuring 7 mils in diameters and depth 0.011 mils were introduced to the bearings using spark erosion method.

Following four Vibration signals were acquired by accelerometer that was stud mounted on the bearing housing:

1. Bearing in normal condition,

2. Bearing with Outer Race fault,

3. Bearing with Inner Race fault, and

4. Bearing with Roller fault.

The shaft was made to rotate at $30 \mathrm{~Hz}$ and vibration signals were collected at sampling rate of $62.5 \mathrm{KSa} / \mathrm{s}$. The signals were collected for duration of $2 \mathrm{~s}$. The instruments used for the experiments include a piezoelectric accelerometer, a charge amplifier, a Stroboscope for speed calibration and a digital data recorder system, for acquiring and storing the vibrations signals. The ED and WA using Continuous Wavelet Transform were performed in Matlab environment.

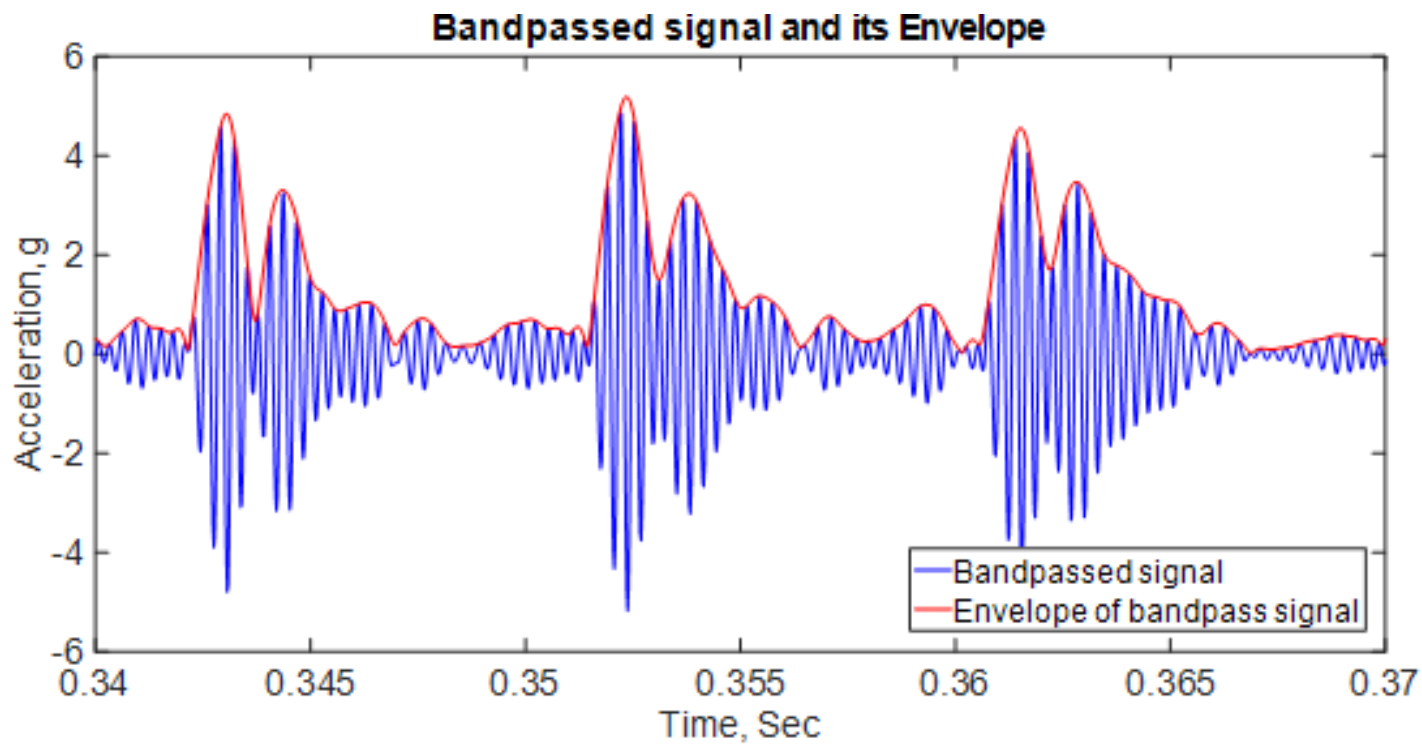

Figure 6: Band passed signal and its envelope 
International Journal of Science and Research (IJSR)

ISSN (Online): 2319-7064

Index Copernicus Value (2015): 78.96 | Impact Factor (2015): 6.391

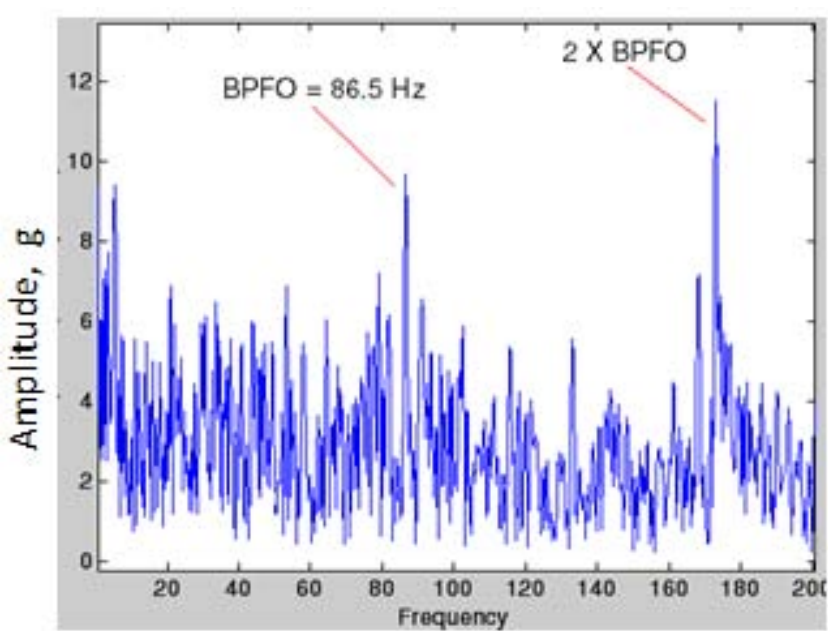

Figure 7: Spectrum of envelope of bearing with outer race fault

Experiment A variety of artificially fault induced ballbearing type MB 204 was used. The types of faults included a defective outer-race, a defective inner-race, and a defective ball. Although the motor was set to rotate at $30 \mathrm{~Hz}$, the actual rotating speed monitored by the stroboscope was found to be $28.85 \mathrm{~Hz}$. The BCF was calculated using Eqs. (1) to (3), the geometric parameters of the bearings are $d$ $=0.3125^{\prime}, \mathrm{D}=1.319^{\prime}, \mathrm{n}=8$ and $\varnothing \sim 2^{\circ}$. The calculated BCF for each type of fault are presented in Table 1. It also shows the time period between the impacts (inverse of BCF) and number of sample points between these impacts, which is equal to time period multiplied by sampling frequency.

\section{Envelope Detection Results}

Fig. 4 shows the simple spectrum of vibrations recorded for bearing with defective outer-race and Fig. 5 shows the spectrum with outer race fault zoomed near low frequency region where the BCFs are likely to be found, the peak at $\mathrm{BCF}$ for outer race defect $\mathrm{BPFO}=86.5 \mathrm{~Hz}$ is hardly discernible even in zoomed spectrum and is most likely to be missed out in presence of more noise. Further, the peaks are so small that trending of amplitude is also quite difficult, thus it is evident that simple spectrum analysis is not a suitable technique for bearing faults analysis.

To implement ED, the center frequency of bandpass filter should be selected to coincide with the center frequency of the resonance to be studied, which generally falls in the range 10 to $50 \mathrm{kHz}$ [3]. In Fig. 4 resonance are visible at 8.8 $\mathrm{kHz}, 17.5 \mathrm{kHz}, 22.5 \mathrm{kHz}$ and $28.3 \mathrm{kHz}$ so the center frequency of the bandpass filter was set to each of these frequencies and results were analysed. It was found that best results were obtained when bandpass filter's central frequency was set to $17.5 \mathrm{kHz}$.

The vibration signal from bearing with defect on outer race was band-passed and the envelope of the band-passed signal was extracted using the Hilbert transform method. The bandpassed signal and the envelope of band-passed signal that was extracted is shown in Fig. 6. It is evident from Fig. 6 that Hilbert transform method has been able extract true envelope of the band-passed signal.

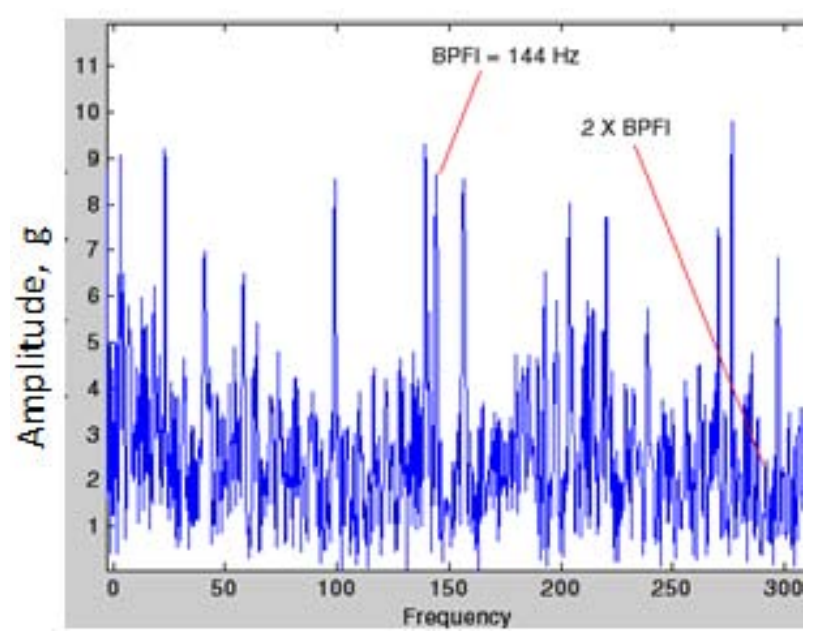

Figure 8: Spectrum of envelope of bearing with inner race fault

The spectra of the envelope for various types of bearing defects obtained by Hilbert transform method are shown in Figs. 7 to 9. Fig. 7 shows the spectrum of the enveloped signal for bearing with outer race defect. From the spectrum plot the frequency of impact (BPFO) at $86.6 \mathrm{~Hz}$ and its second harmonic at $173 \mathrm{~Hz}$ can be clearly recognized. Hence, identification of defect as outer-race defect can be made. In Fig. 8 the impact repetition frequency of inner race fault (BPFI) at $144 \mathrm{~Hz}$ can be recognized however; its second harmonic at $288 \mathrm{~Hz}$ is not very evident. But still identification of fault as inner race fault can be made. However, the inspection of Fig. 9. that is the envelope spectrum for ball defect cannot be identified as rolling element fault since the peak at BPFR $(108 \mathrm{~Hz})$ as well as its second harmonic at $216 \mathrm{~Hz}$ are not prominent. They are submerged in random noise and spectrum peaks due to other phenomenon.

From above results, it is evident that the ED technique is able to reveal the defects but the results are not consistent for all types of defects. The technique is able to give excellent results for bearings having outer race defect. However, detection of roller and inner race defect may not be as definitive.

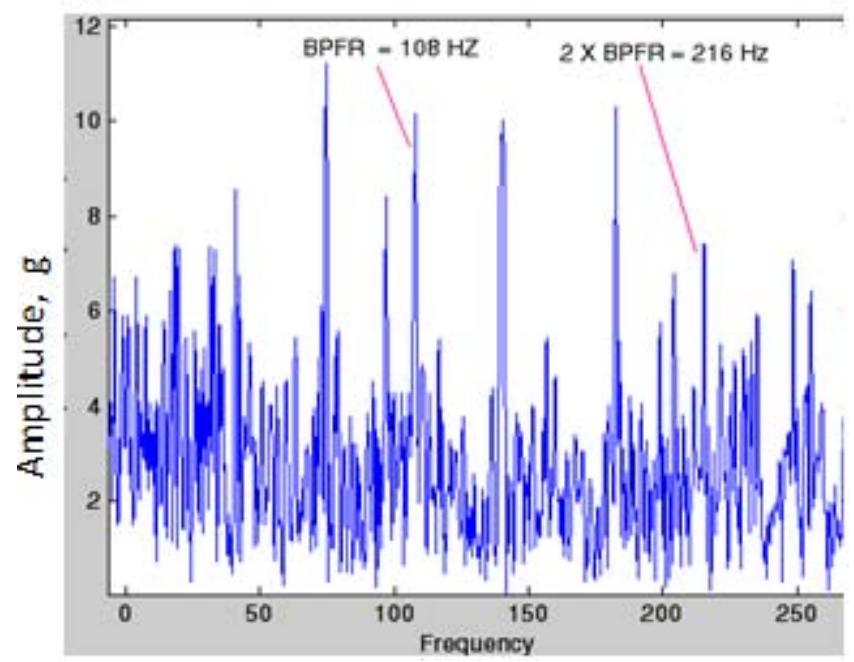

Figure 9: Spectrum of envelope of bearing with fault on rolling element 


\section{International Journal of Science and Research (IJSR) \\ ISSN (Online): 2319-7064}

Index Copernicus Value (2015): 78.96 | Impact Factor (2015): 6.391

\section{Results of Fault Diagnosis Using Wavelet Analysis}

In this section a simple CWT based wavelet analysis method to identify bearing faults is described. This method do not involve the elaborate signal processing that is required to implement ED. The CWT based WA is a simple visual inspection method and it does not require the analyst to have a lot of experience in Fault diagnosis or signal processing.

Some typical results obtained by doing CWT analysis of vibration signals obtained from the bearings running at $28.85 \mathrm{~Hz}$ are shown here. The time-scale distribution diagrams generated by CWT for bearings running under normal condition and with outer-race fault, inner-race fault and rolling element fault are shown in Figs. 10 to 13 respectively. These four figures give a good qualitative assessment of bearing condition.

The plots in Fig 10 to 13 are the of CWT coefficients plotted on a time (samples) \& scale (frequency) grid, the brightness of colour indicates the amplitude at respective point on the sample - scale grid. The plots are 8192 samples each that are equal to 131 milliseconds. The parameter scale as explained earlier may be considered as inverse of frequency. The low scales represents high frequency band and high scales represents low frequencies. When the bearing is operating in normal condition, the energy level in the low frequency range, where most of the macro-structural vibrations are located, should be low. This can be noticed in Fig. 10, where dark color in the low frequency range (scales 256 to 4096) indicates low energy content and suggest that bearing is healthy.

On the other hand high if high energy content is noticed in low frequency range where the bearing characteristic frequency lies, it indicates towards defective bearing. In Fig. 11, where an outer-race fault exists in the bearing, the energy levels of vibrations in the low frequency range are high as highlighted by bright colored contours in low frequency range.

Another evidence of occurrence of a fault could be obtained by inspecting the energy level at the high frequency range, where the bearing excitation range is located i.e. scales 6 to 2 . The impacts caused by passage of rolling element over the defect causes an impact which excites the bearing resonant frequency. The bearing structural frequency lies in high frequency range about $10 \mathrm{kHz}$ to $50 \mathrm{kHz}$. Due to inherent damping present in system the impacts causes' short duration high frequency damped oscillations. Figs. 12 and 13 show the high-energy impacts generated by the defects in the inner-race and the roller respectively. The Impacts are indicated by appearance of strips of bright color (high energy) occurring at regular interval.

It is always desirable to know the cause of a fault. The length of time interval of the impacts is inversely proportional to the Bearing Characteristic Defect (BCF), the cause of the fault can be determined by measuring the time interval of each impact at the high frequency bearing

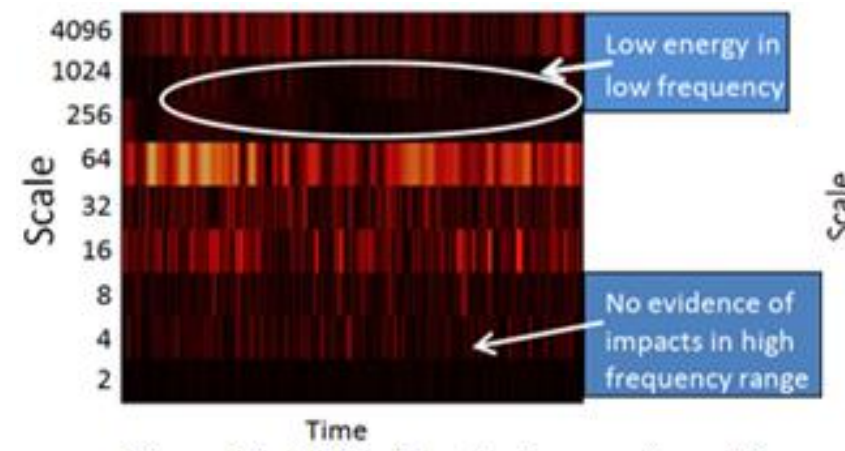

Figure 10: CWT of bearing in nomal condition

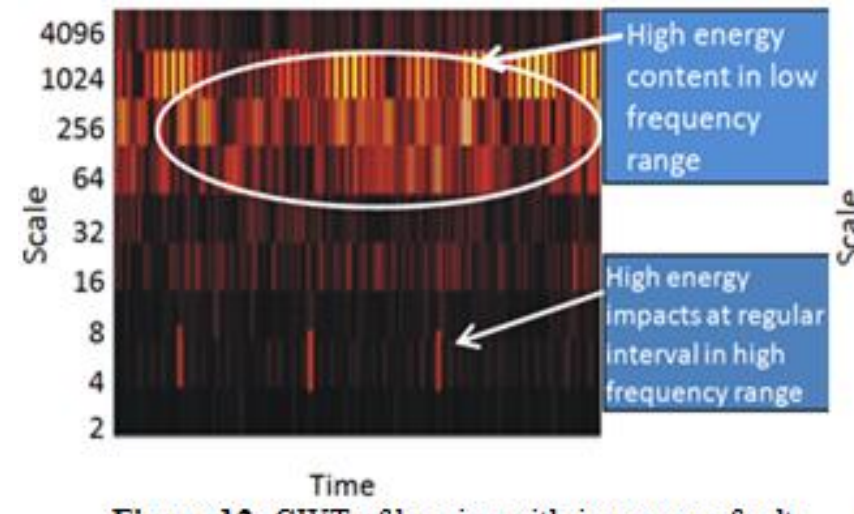

Figure 12: CWT of bearing with inner race fault

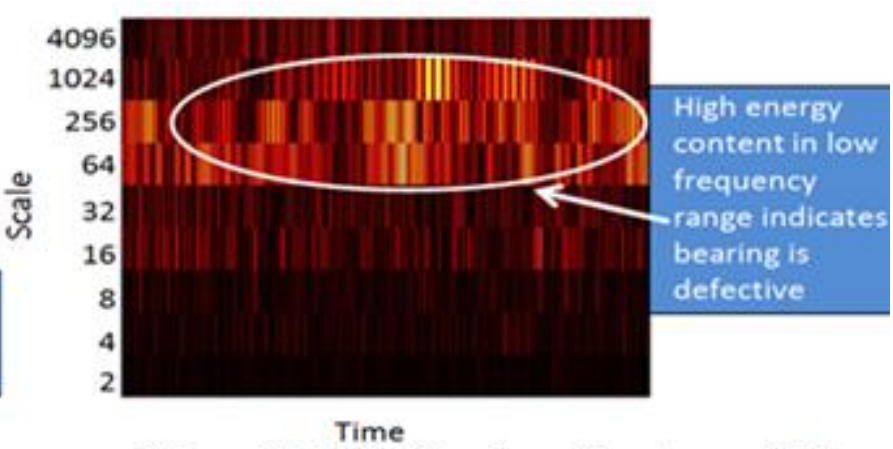

Figure 11: CWT of bearing with outer race fault

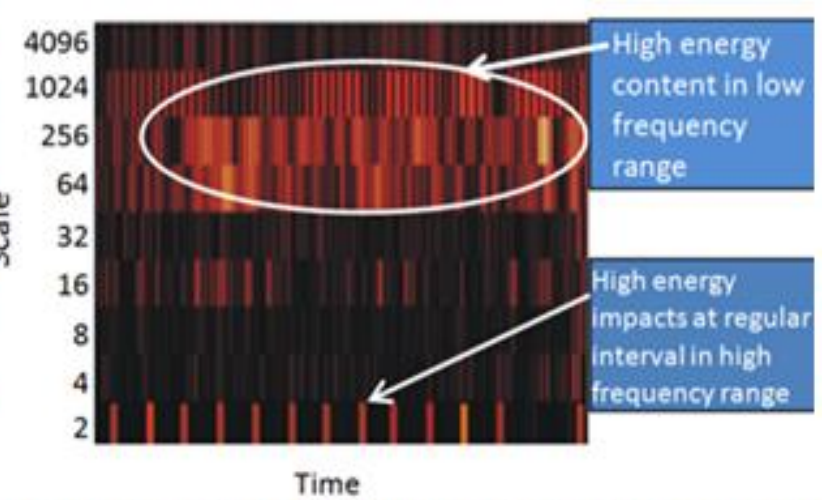

Figure 13: CWT of bearing with fault on rolling element 


\section{International Journal of Science and Research (IJSR)

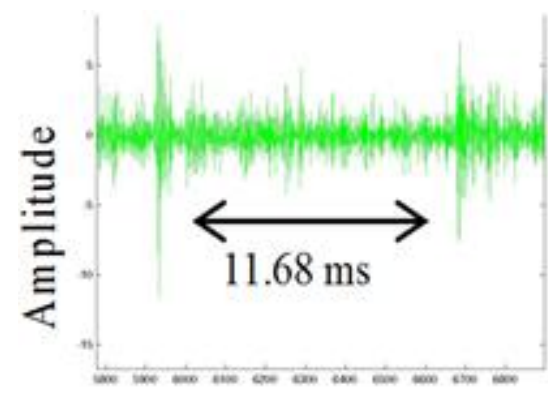

(a)
Samples

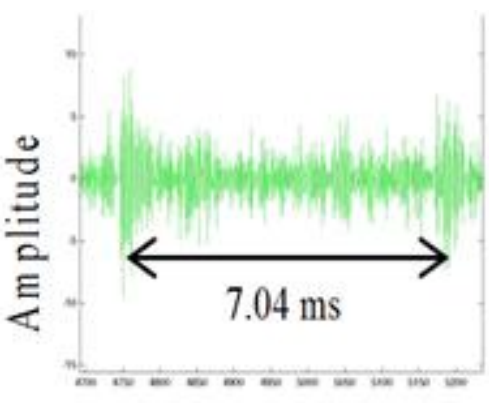

(b)

\section{Samples}

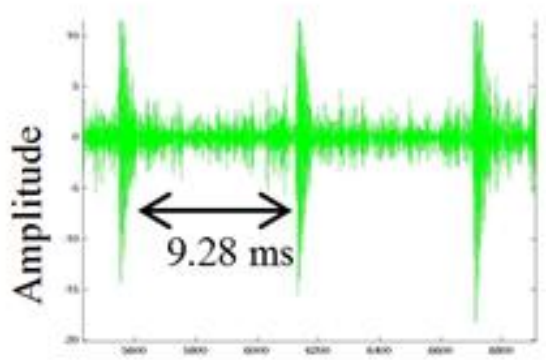

(c)

\section{Samples}

Figure 14: CWT coefficients at scale 2 (a) Bearing with outer-race defect (b) Bearing with inner-race defect (c) Bearing with ball defect

excitation range in the time-frequency distribution diagrams and then comparing the time intervals listed in Table I. To let the operator performs the visual inspection of the time intervals of impacts, the results of WA are displayed as plot of CWT coefficients in scale 2 representing central frequency about $20 \mathrm{kHz}$ is presented in Fig. 14 (a) to (c) for bearing having outer race defect, inner race defect and ball fault respectively. The scale 2 was selected since the bearing excitation range is embedded in the high frequency range.

In the plots of CWT coefficients at scale 2 shown in Fig. 14, the impacts are clearly identifiable as well as the time period between the impacts can also be easily measured. The time intervals of impacts in Figs. 14 (a), (b) and (c) are estimated as $7.04 \mathrm{~ms}, 11.68 \mathrm{~ms}$ and $9.28 \mathrm{~ms}$ respectively. These intervals correspond to the inverse of the calculated BPFO (86.49), BPFI (144.3 Hz), and BPFR (107.9 Hz), corresponding to the defects of inner-race, outer-race and roller respectively as given in Table 1 . The procedures of using WA in the fault diagnosis of rolling element bearings can be divided into two stages. In the first stage, if highenergy are observed in low frequency BCF range of the time-scale distribution diagram, then faults may be occurring in the bearing. Essential evidence to prove the existence of faults may be obtained by inspecting whether a high-energy impact is evident in low scales of time-scale distribution diagram. If the cause of fault must be identified, then the second stage of inspecting the time interval of impacts in the high frequency bearing excitation range should be performed.

\section{Conclusions}

Both the methods of bearing fault detection that are analysed in this paper i.e. Envelope Detection and Wavelet analysis with CWT are effective to identify bearing defects. However, the faults caused by defective inner-race and rollers are more difficult to be identified by ED technique. Whereas; by using the time-frequency distribution diagrams provided by CWT, the high-energy impacts caused by innerrace and ball defects can be easily identified in the high frequency range. Thus CWT allows for identification of all three types of bearing faults.

Wavelet analysis using CWT provide good resolution in frequency at the low frequency range, and fine resolution in time at the high frequency range. Such a multi-resolution capability is an advantage for vibration-based machine fault diagnosis. The outer race faults can be detected by both CWT as well as by ED. However, wavelet analysis by using CWT is better than the envelope detection method in detection of inner race faults and fault of rolling element. CWT method is considered to be superior to ED. Further, CWT based wavelet analysis described in the paper is a simple visual inspection method and it does not require the analyst to have a lot of experience in Fault diagnosis.

\section{Nomenclature}

$\begin{array}{ll}\mathrm{d} & \text { Ball diameter. } \\ \mathrm{D} & \text { Bearing pitch diameter. } \\ \mathrm{H}(\omega) & \text { Frequency response function of Hilbert Transformer. } \\ \mathrm{n} & \text { Number of balls. } \\ \mathrm{s} & \text { Scale parameter. } \\ \mathrm{V}(\mathrm{t}) & \text { Envelope. } \\ W_{\Psi}(\tau, s) & \text { CWT coefficient. } \\ \mathrm{x}(\mathrm{t}) & \text { Time domain signal. } \\ \hat{\mathrm{x}}(\mathrm{t}) & \text { Hilbert transform of } \mathrm{x}(\mathrm{t}) . \\ \mathrm{x}_{+}(\mathrm{t}) & \text { Analytic signal. } \\ \beta & \text { Bearing contact angle. } \\ \tau & \text { Translation parameter. } \\ \psi & \text { Mother wavelet. } \\ \Psi_{\tau, g}(t) & \text { Scaled and shifted version of mother wavelet. }\end{array}$

\section{References}

[1] Randall RB, Antoni J. Rolling element bearing diagnostics - a tutorial. Mechanical systems and signal processing. $2011 \mathrm{Feb} 28 ; 25(2): 485-520$.

[2] Norton MP, Karczub DG. Fundamentals of noise and vibration analysis for engineers. Cambridge university press; 2003 Oct 16.

[3] Tandon N, Choudhury A. A review of vibration and acoustic measurement methods for the detection of defects in rolling element bearings. Tribology international. 1999 Aug 31;32(8):469-80.

[4] McFadden PD, Smith JD. Vibration monitoring of rolling element bearings by the high-frequency resonance technique - a review. Tribology international. 1984 Feb 1;17(1):3-10.

[5] Wang D, Miao Q, Fan X, Huang HZ. Rolling element bearing fault detection using an improved combination of Hilbert and wavelet transforms. Journal of 
Mechanical Science and Technology. 2009 Dec 1;23(12):3292-301.

[6] Peng YH, Yam R. Wavelet analysis and envelope detection for rolling element bearing fault diagnosistheir effectiveness and flexibilities. Journal of Vibration and Acoustics, Transactions of the ASME. 2001;123:303-10.

[7] Lou X, Loparo KA. Bearing fault diagnosis based on wavelet transform and fuzzy inference. Mechanical systems and signal processing. 2004 Sep 30;18(5):107795.

[8] Peng ZK, Peter WT, Chu FL. A comparison study of improved Hilbert-Huang transform and wavelet transform: application to fault diagnosis for rolling bearing. Mechanical systems and signal processing. 2005 Sep 30;19(5):974-88.

[9] Yavanarani K, Raj GS, Christabel SS, Vijayaraghavan J. Multi resolution analysis for bearing fault diagnosis. InFrontiers in Automobile and Mechanical Engineering (FAME), 20102010 Nov 25 (pp. 322-327). IEEE.

[10] Carcaterra A, Sestieri A. Complex envelope displacement analysis: a quasi-static approach to vibrations. Journal of Sound and Vibration. 1997 Mar 27;201(2):205-33.

[11] Mallat SG. A theory for multiresolution signal decomposition: the wavelet representation. IEEE transactions on pattern analysis and machine intelligence. $1989 \mathrm{Jul} ; 11(7): 674-93$.

[12] Mallat S. A wavelet tour of signal processing. Academic press; 1999 Sep 14.

\section{Author Profile}

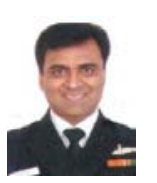

Sunil Tyagi received the B.E. and M.E., degrees from GB Pant University, Pant Nagar, India, in 1991 and Pune University, India in 2002 respectively. He was commissioned in Engineering Branch of Indian Navy in 1992 and presently serving Indian Navy in the rank of Commander. He has over eleven years of experience on operations and design of onboard marine machinery and about five years of teaching. $\mathrm{He}$ is pursuing doctoral programme from mechanical engineering department of DIAT, Pune. His main areas of research interest are application of soft computing techniques for machinery fault diagnosis. Cdr Tyagi is a member of Institute of Marine Engineers (India) since 2006.

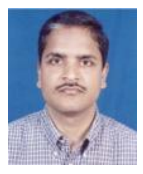

S K Panigrahi received his Bachelor, Master and $\mathrm{Ph}$ $\mathrm{D}$ degree in Mechanical Engineering from Veer Surendra Sai Technical University (VSSUT), formerly known as University College Engineering (UCE), Burla, National Institute of Technology (NIT), Rourkela and Indian Institute of Technology, Kharagpur in 1992, 1996 and 2007. He has held many positions such as Associate Professor, Assistant professor, Assistant Professor (Selection Grade), Lecturer (Senior Scale) and Lecturer in different University / Institution within the country prior to his joining at Defence Institute of Advanced Technology (Deemed University), Pune. He has also worked as an International Visiting Academic with University of New South Wales at the Australian Defence Force Academy (ADFA). Presently, he is working as Head of Mechanical Engineering Department at DIAT (DU), Pune. 